\title{
Portomesenteric Venous Thrombosis with Bowel Ischemia after Laparoscopic Sleeve Gastrectomy
}

\author{
Mubarak Alkandari ${ }^{1}$, Samir Abdulaziz ${ }^{2}$, Mohamed Alsulimy $^{3}$, Nagy Ismaeil ${ }^{4}$, Mohamed Mohsen $^{5}$
}

\begin{abstract}
Laparoscopic sleeve gastrectomy has become an increasingly popular surgical option for morbidly obese patients. Portomesenteric venous thrombosis, as a complication of laparoscopic sleeve gastrectomy, has been rarely reported. We report one case of thrombosis of the portal vein and the superior mesenteric vein after sleeve gastrectomy. It is confirmed by CT scan. Thrombosis of the portomesenteric veins after bariatric surgery is a diagnosis that one should know how to raise in front of any postoperative abdominal pain. An obese patient with a history of thrombosis should receive a complete etiology of these thromboses before bariatric surgery. Abnormal blood dirt, an active smoking fat woman having oral contraceptive, or a patient with a history of recurrent venous thrombosis may be a relative contraindication against a complex bariatric surgery with digestive bypass.

Keywords: Bowel ischemia, Diagnostic laparoscopy, Laparoscopic sleeve gastrectomy.

World Journal of Laparoscopic Surgery (2019): 10.5005/jp-journals-10033-1385
\end{abstract}

\section{INTRODUCTION}

Bariatric surgery is being used with increased frequency for effective weight loss in patient with morbid obesity. ${ }^{1}$ However, although rare, life-threatening complications such as mesenteric ischemia and pulmonary embolism occur. ${ }^{2}$ The incidence of deep vein thrombosis (DVT) after bariatric surgery varies from 1.2 to $1.6 \% .^{3}$ Portomesenteric venous thrombosis (PMVT) is a rare vascular event but is associated with high mortality rates (20-45\%). ${ }^{3,4}$

\section{Case Description}

A 35-year-old patient underwent a laparoscopic sleeve gastrectomy for the treatment of obesity (BMI $41 \mathrm{~kg} / \mathrm{m}^{2}$ ). The patient was not a smoker and she was not having any chronic debilitated diseases. The surgical procedure was simple with an operative time of 45 minutes. The immediate postoperative course was simple and uneventful, and the hospitalization ended on the $3 \mathrm{rd}$ day. The patient was again admitted to emergency on the 14th postoperative day for severe epigastric abdominal pain associated with nausea and persistent repeated vomiting of 2 days' duration. On examination, she was vitally stable with BP: $110 / 60 \mathrm{mmHg}$, temp.: $36.8^{\circ} \mathrm{C}$, and pulse: $90 \mathrm{~b} / \mathrm{m}$. The abdomen was tender with guarding, while the patient was tachycardic but febrile. X-ray abdomen showed dilated small bowel loops. The investigations were found without abnormalities apart from positive ketonuria and high serum lactate. The computed tomography (CT) found a thrombosis of both the portal vein (Fig. 1) and the superior mesenteric vein (Fig. 2) associated to the thick enedjejunal loop with extensive collection. An emergency laparoscopy was done after anesthesia consultation and informed consent from the patient as regard to risk, benefits, and outcomes of the surgery that revealed intra-abdominal free fluid and a gangrenous small bowel segment and then conversation laparotomy was performed. A $100 \mathrm{~cm}$ of ischemic small bowel segment that began at the $20 \mathrm{~cm}$ from the Treitz ligament was resected (Fig. 3). The gastrointestinal continuity was provided by an end-to-end anastomosis. The patient's postoperative course was uneventful. On the 4 th postoperative day, oral fluids were started.
${ }^{1-5}$ Department of Surgery, Al-Sabah Hospital, Kuwait

Corresponding Author: Mohamed Mohsen, Department of Surgery, Al-Sabah Hospital, Kuwait, Phone: +965 60463440, e-mail: mohsenroom@yahoo.com

How to cite this article: Alkandari M, Abdulaziz S, Alsulimy $M$, et al. Portomesenteric Venous Thrombosis with Bowel Ischemia after Laparoscopic Sleeve Gastrectomy. World J Lap Surg 2019;12(3):135-137.

Source of support: Nil

Conflict of interest: None

He was discharged on the 7th postoperative day. Pre- and postoperative activated partial thromboplastin time (aPTT) and international normalized ratio (INR) values were observed; other blood tests, protein C and S deficiency, and phospholipid enzymes assessment had been requested. The patient underwent in the postoperative hospitalization period low molecular weight

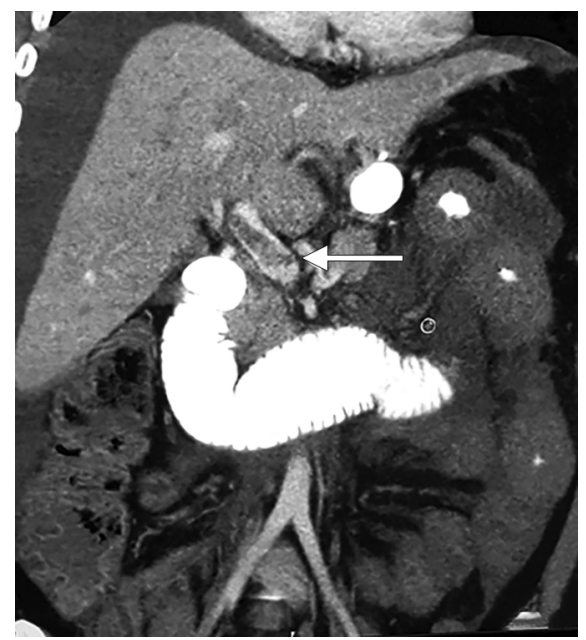

Fig. 1: Abdominal CT scan with contrast showing portal vein thrombosis (white arrow) 


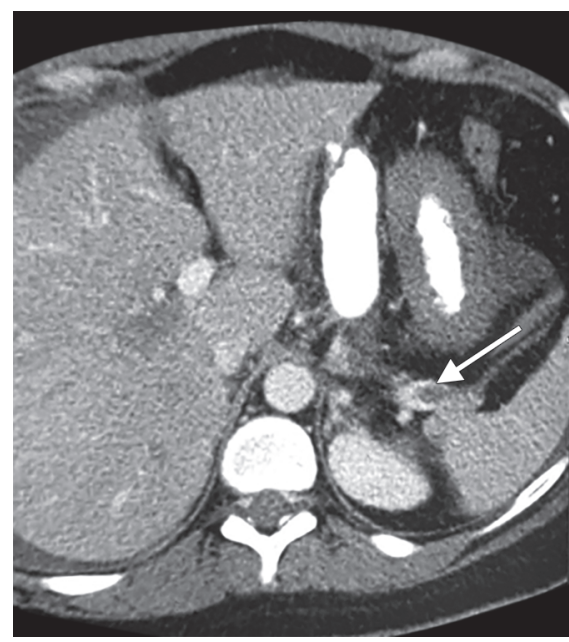

Fig. 2: Abdominal CT scan with contrast showing superior mesenteric vein thrombosis (white arrow)

heparin (LMWH) (enoxaparin $80 \mathrm{mg}$ twice/daily) followed by oral anticoagulation after discharge (warfarin $5 \mathrm{mg} /$ day) for up to 6 months. Histopathological examination revealed gangrenous necrosis with mesenteric vascular thrombosis in the resected jejunal segment.

\section{Discussion}

The most frequent complications following sleeve gastrectomy are fistula and hemorrhage. Thrombosis of the superior mesenteric vein is exceptional, potentially severe. ${ }^{5}$ In this case described above, the diagnosis was made on the 14th postoperative day. There are multiple risk factors such as genetic predisposition and hematological factors (factor $\mathrm{V}$ Leiden deficiency, protein $\mathrm{C}$ and $S$ deficiency), malignancy, immobilization, varicose veins, atrial fibrillation, and venous stasis due to intra-abdominal pressure, intraoperative manipulation, and/or damage at the splanchnic endothelium, which can lead to PMVT. Diabetes mellitus (DM) is an important causing factor in the development of atherothrombosis by dysregulation of several signaling pathways resulting in enhanced adhesion, activation, and aggregation of the platelets. ${ }^{6}$ Overt hyperthyroidism is also associated with venous thrombosis particularly in cerebral and splanchnic veins. ${ }^{7}$ The clinical signs of mesenteric venous ischemia are variable and nonspecific. In the presence of abdominal pain of unknown etiology, we shall know how to suggest the diagnosis of a portal vein thrombosis. In case of intestinal ischemia, pain, which is always present, contrasts the absence of physical signs. ${ }^{8,9}$ It can be associated with nausea, vomiting, diarrhea, and high or low gastrointestinal hemorrhage. ${ }^{10}$ The presumptive diagnosis is often that of perforated ulcer or acute pancreatitis. ${ }^{11}$ Biology may not show leukocytosis in half of patients. ${ }^{10}$ In obese patients, the reference radiological examination is the CT with vascular injection. ${ }^{12,13}$ Familiarity with this dangerous entity is important. Prompt diagnosis and care, initiated by a high index of suspicion, is crucial. The treatment of acute intestinal ischemia of the venous origin has evolved in the last years..$^{14}$ It is now mainly medical; in case of early diagnosis and an abdomen that is "not acute" and presented no infarction, two nonoperative treatments may be considered: thrombolysis and systemic heparin. ${ }^{8,14}$ Surgical exploration by laparoscopy is useful in acute abdomens but remains of rare use and is still being discussed in this

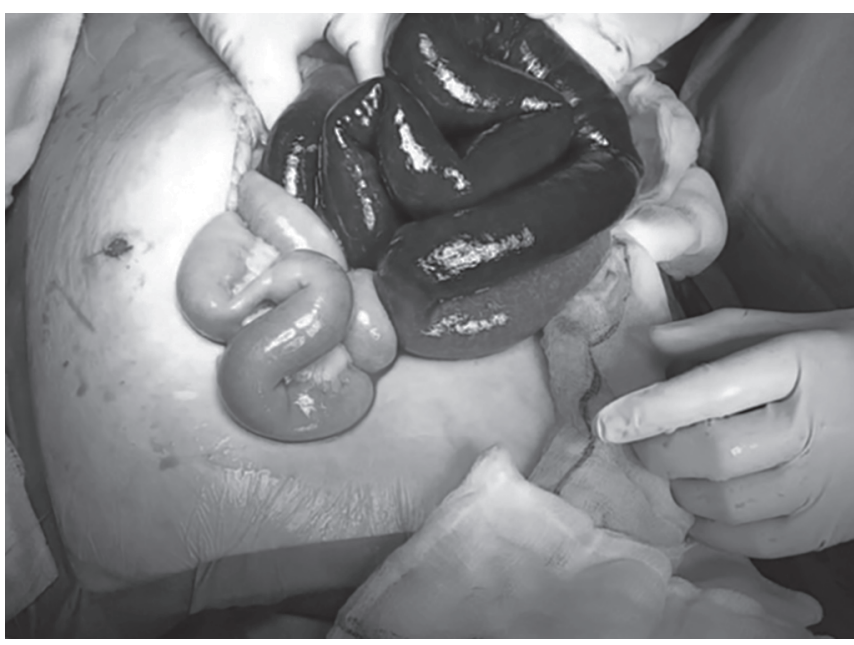

Fig. 3: Small bowel gangrenous segment

indication because the hypertension associated within sufflation could theoretically worsen venous ischemia. ${ }^{15}$ Surgical exploration consents the lesions assessment with two possible outcomes: (1) in cases of localized intestinal necrosis, treatment consists of a resection and immediate restoration of digestive continuity; (2) when the ischemic or infarcted intestine segment is extended, the limits of resection are difficult to predict. In all cases, the resection should be efficient to avoid "short bowel syndrome." Some teams are partisans of a resection followed by a gastrointestinal bypass, associated with an immediate heparin treatment ensued by a second laparotomy $12-24$ hours later. ${ }^{10}$

\section{Conclusion}

Portomesenteric vein thrombosis is a complication that has potentially life- threatening consequences following laparoscopic bariatric surgery. It should be of clinical suspicion as it presents with nonspecific symptoms. In cases with nonspecific abdominal pain after bariatric surgery, possible portal vein thrombosis (PVT) diagnosis should be kept in mind, and necessary radiological procedures should be used for early diagnosis and treatment.

\section{References}

1. Çetinkünar S, Erdem H, Aktimur R, et. al. The effect of laparoscopic sleeve gastrectomy on morbid obesity and obesity related comorbidities: a cohort study. Ulus Cerrahi Derg 2015;31(4):202. DOI: 10.5152/UCD.2015.2993.

2. Bajardi G, Ricevuto G, Mastrandrea G, et al. Postoperative venous thromboembolism in bariatric surgery. Minerva Chir 1993;48(10):539.

3. Acosta S, Alhadad A, Svensson P, et al. Epidemiology, risk and prognostic factors in mesenteric venous thrombosis. Br J Surg 2008;95(10):1245-1251. DOI: 10.1002/bjs.6319.

4. Westling A, Bergqvist D, Bostrom A, et al. Incidence of deep venous thrombosis in patients undergoing obesity surgery. World J Surg 2002;26(4):470. DOI: 10.1007/s00268-001-0251-9.

5. Swartz DE, Felix EL. Acute mesenteric venous thrombosis following laparoscopic Roux-en-Y gastric bypass. JSLS 2004;8(2):165-169.

6. Vazzana N, Ranalli P, Cuccurullo C, et al. Diabetes mellitus and thrombosis. Thromb Res 2012;129(3):371-377. DOI: 10.1016/ j.thromres.2011.11.052.

7. Franchini M, Lippi G, Targher G. Hyperthyroidism and venous thrombosis: a casual or causal association? A systematic literature 
review. Clin Appl Thromb Hemost 2011;17(4):387-392. DOI: 10.1177/1076029610364521.

8. Cohen J, Edelman RR, Chopra S. Portal vein thrombosis: a review. Am J Med 1992;92(2):173-182. DOI: 10.1016/0002-9343(92)90109-O.

9. Abdu RA, Zakhour BJ, Dallis DJ. Mesenteric venous thrombosis 1911 to 1984. Surgery 1987;101(4):383-388.

10. Rhee RY, Gloviczki P, Mendonca CT, et al. Mesenteric venous thrombosis: still a lethal disease in the 1990s. J Vasc Surg 1994;20(5):688-697. DOI: 10.1016/s0741-5214(94)70155-5.

11. Case records of the Massachusetts General Hospital. Weekly clinicopathological exercises. Case 51-1984. A 45-year-old man with abdominal pain and abnormal CT scan. N Engl J Med 1984;311(25): 1622-1628. DOI: 10.1056/NEJM198412203112508.
12. Parvey HR, Raval B, Sandler CM. Portal vein thrombosis: imaging findings. AJR Am J Roentgenol 1994;162(1):77-81. DOI: 10.2214/ ajr.162.1.8273695.

13. Verbanck JJ, Rutgeerts LJ, Haerens $M H$, et al. Partial splenoportal and superior mesenteric venous thrombosis. Early sonographic diagnosis and successful conservative management. Gastroenterology 1984;86(5 Pt 1):949-952.

14. Bradbury AW, Brittenden J, McBride K, et al. Mesenteric ischaemia: a multidisciplinary approach. Br J Surg 1995;82(11):1446-1459. DOI: 10.1002/bjs.1800821105.

15. Henry C, Smadja C, Vons C, et al. Results of laparoscopic treatment of abdominal emergencies. Ann Chir 1998;52(3):223-228. 